\title{
Holistically assessing and improving the sustainability of aquaculture development in China
}

\section{Shuanglin Dong ( $\nabla$ dongsl@ouc.edu.cn )}

Ocean University of China

\section{Yun-wei Dong}

Ocean University of China

Johan Verreth

Wageningen University and Research

\section{Yngvar Olsen}

Norwegian University of Science \& Technology

Wen-jing Liu

Ocean University of China

\section{Qi-zhi Fang}

Ocean University of China

\section{Ling Cao}

Shanghai Jiao Tong University

\section{Yan-gen Zhou}

Ocean University of China

Li Li

Ocean University of China

Jing-yu Li

Ocean University of China

\section{Yong-tong Mu}

Ocean University of China

\section{Patrick Sorgeloos}

Ghent University

\section{Article}

Keywords: Aquaculture, China, aquaculture production systems, ecological intensification

Posted Date: October 1st, 2020

DOI: https://doi.org/10.21203/rs.3.rs-75225/v1 
License: (c) (i) This work is licensed under a Creative Commons Attribution 4.0 International License. Read Full License 


\section{Holistically assessing and improving the sustainability of aquaculture development in China}

Shuang-lin Dong ${ }^{1.2} \llbracket *$, Yun-wei Dong ${ }^{1 *}$, Johan Verreth ${ }^{3}$, Yngvar Olsen ${ }^{4}$, Wen-jing $\mathrm{Liu}^{5}$, Qi-zhi Fang ${ }^{5}$, Ling $\mathrm{CaO}^{6}$, Yan-gen Zhou ${ }^{1}$, Li Li $i^{1}$, Jing-yu Li ${ }^{1}$, Yong-tong $\mathrm{Mu}^{1}$, Patrick Sorgeloos ${ }^{7 凶}$

1. Key Laboratory of Mariculture (Ocean University of China), Ministry of Education, Ocean University of China, Qingdao, P. R. China

2. Function Laboratory for Marine Fisheries Science and Food Production Processes, Qingdao National Laboratory for Marine Science and Technology, Qingdao, P. R. China

3. Aquaculture \& Fisheries Group, Wageningen University and Research, Wageningen, the Netherlands

4. Department of Biology, Norwegian University of Science \& Technology, Trondheim, Norway

5. School of Mathematical Science, Ocean University of China, Qingdao, P. R. China

6. School of Oceanography, Shanghai Jiao Tong University, Shanghai, P. R. China

7. Laboratory of Aquaculture \& Artemia Reference Center, Ghent University, Gent, Belgium

$\square_{T}$ o whom correspondence may be addressed. Email: dongsl@ouc.edu.ch; Patrick.Sorgeloos@UGent.be.

*S.L.D. and Y.W.D. contributed equally to this work. 


\section{Abstract}

Aquaculture is the fastest growing food production industry in terms of annual growth rate and has become an important contributor in supplying essential macro-and micro-nutrients for the global population. However, there is a great deal of uncertainty in its further development due to the high and increasing pressure of environmental challenges and of resource constraints. To achieve overall sustainable development of the aquaculture sector in China, the dominant producer of farmed seafood in the world, the priority is to clarify the sustainability of major aquaculture production systems (APSs) and to seek a holistic approach to improve their sustainability. Here the sustainability of ten major types of APSs in China, was evaluated comparatively by three objective and subjective methods involving eleven social, economic, environmental, and resource criteria. Accordingly, ecological intensification of aquaculture systems (ELIAS), integrating anthropogenic inputs with aquaculture ecosystem services, is proposed to improve the sustainability of these APSs. ELIAS that should be widely adopted by the multiple stakeholders provides a feasible solution to realize the goals of increasing aquaculture outputs with less negative environmental influence while saving energy at the same time. 
During the past decades aquaculture was the fastest growing food production industry in terms of annual growth rate, far outpacing human population growth ${ }^{1}$. Therefore, it might continue to provide important guarantees for food security, with a supply of high-quality macro- and micronutrients ${ }^{2}$. Increasing the consumption of farmed seafood instead of terrestrially farmed animal meat could potentially reduce the amount of land required for growing feed crops for a global population of nine billion people by $2050^{3}$.

The development of world aquaculture in the past decades has been achieved mainly through intensification of production systems $s^{4,5}$. Globally, simplistic intensification of a production ecosystem may yield a high and predictable production in a short term, however, it can potentially face pervasive risks in a longer term ${ }^{4-6}$. Hereby, sustainable intensification was proposed to achieve food security, economic, social, and environmental goals of food production systems ${ }^{7,8}$. Like other food production systems, aquaculture also underwent an intensification process in the past decades, and there is a great deal of uncertainty in projected growth rates due to the high and increasing pressure of environmental challenges and resource constraints ${ }^{2,4}$. The future contribution of aquaculture to world food security depends largely on its sustainability.

In the past decade, sustainable development of aquaculture has been studied from many perspectives, e.g. environmental impacts ${ }^{8}$, resource constraints $^{9,10}$, certification ${ }^{11}$, markets ${ }^{12}$, finance ${ }^{13}$, society $^{14}$, and policies ${ }^{15}$. However, the sustainability of different aquaculture production systems (APSs) has seldomly been evaluated involving environmental, economic, resource and social criteria due to the absence of enough objective measures for all these criteria, just as is the case for animal aquaculture systems in USA ${ }^{16}$.

Although disease prevention ${ }^{17}$, improvements of genetic stocks ${ }^{18}$, feeds ${ }^{19}$ and farm management ${ }^{20}$ can improve economic benefits, relieve resource constraints, and reduce the negative environmental impacts of APSs, the comprehensive benefits of aquaculture production ecosystems are largely determined by their trophic structures and functions ${ }^{21}$, which should be optimized primarily. Humankind is facing the triple crucial challenges of population growth, environmental pollution, and global climate change $\mathrm{e}^{3,22}$. 
Therefore, a holistic approach is urgently needed to consider increasing production, reducing waste discharge and carbon footprint of APSs simultaneously. Although several creative aquaculture systems, e.g., recirculating aquaculture systems (RAS), and bioflocs, have been invented ${ }^{4,23}$, most of them cannot meet the three aforementioned requirements at the same time.

In this article the sustainability of ten major kinds of APSs in China mainland, in which fifty-eight percent of the world aquaculture production was produced ${ }^{2}$ in 2018, was evaluated comparatively by three methods, i.e. a classified aggregation based on objective data, analytical hierarchy process $(\mathrm{AHP})^{24}$, and AHP partially based on objective data involving society, economy, environment, and resources. Furthermore, a holistic approach of ecological intensification of aquaculture systems (ELIAS), with some feasible suggestions, is proposed to improve the sustainability of future aquaculture developments.

\section{Uncertainty of future aquaculture in China}

World aquaculture production in 2018 was 82.1 million metric tonnes ( $\mathrm{mmt}$, live weight), excluding $30.1 \mathrm{mmt}$ of aquatic plants, with a farmgate value of USD 250.1 billion. It grew on average at 5.3\% per year in the period 2001-2018, whereas the growth was only 4\% in 2017 and 3.2\% in 2018 due to a dramatic slowdown in the growth in China ${ }^{2}$. Because of China's prominence in aquaculture, the uncertainty of China's aquaculture development could has substantial global implications.

For the last 30 years, the aquaculture production (excluding seaweeds) in China has increased by 8.73-times from $5.45 \mathrm{mmt}$ in 1989 to $47.56 \mathrm{mmt}$ in 2018 (Fig. 1a). China's aquaculture is one of the most ecologically efficient industries in the world due to the lower trophic levels of the cultured species ${ }^{2,25}$ (Fig. 1c). However, there is a great deal of uncertainty in China's aquaculture development due to the high and increasing pressure of environmental challenges and of resource constraints. Under these challenges and constraints, the Thirteenth Five-Year Plan for Economic and Social Development of the People's Republic of China (2016-2020) identified that aquaculture policies needed to achieve a sustainable, healthy, and environmental-friendly industry ${ }^{25,26}$. Hereby, FAO estimated that China's aquaculture production would continue to increase under three possible scenarios, i.e. no-plan (without implementation 
of a stringent environmental protection policy), baseline, and full-plan (full implementation of a stringent environmental protection policy), by 36.5\%, 31.1\% and $24.7 \%$ in 2030 over 2016, respectively. Accordingly, the world aquaculture production would increase by $38.3 \%, 36.7 \%$ and $34.8 \%$, respectively ${ }^{26}$.

Aquaculture is performed in a wide range of different production systems farming aquatic plants, herbivores, omnivores, carnivores, filter-feeders as well as deposit-feeders in freshwater or seawater in tanks, ponds, reservoirs, lakes, nearshore and offshore waters. The diversity of China's APSs is one of the highest in the world. Generally, the APSs in China can be divided into ten major types based on feeding strategy, location, and environment (Box 1, Fig. 1b). The future contribution of aquaculture to food security depends largely on the sustainability of various APSs. Sustainability of APSs is described as long-term development integrating four dimensions, i.e. environment, resource, economy, and society, involving eleven criteria (Box 2). If economic and social profits were the main goals for the future aquaculture, aquaculture expansion should be encouraged under a relatively loose environmental protection policy (Fig. 2a). When considering environmental and resource criteria, aquaculture should be constrained in some APSs. Clearly, in light of these criteria, the development of China's aquaculture requires an integrative and systematic evaluation in the context of sustainable development.

\begin{tabular}{|l|}
\hline \multicolumn{1}{|c|}{ Box 1. Aquaculture production systems (APSs) in China } \\
\hline SALA-waterlogged salt-alkali land aquaculture, \\
FAL-fed aquaculture in large inland waters, such as reservoirs and lakes, \\
FAP-fed aquaculture in ponds, \\
FEN-fed nearshore aquaculture, \\
FOA-fed offshore aquaculture, \\
nFAL-non-fed aquaculture in large inland waters, \\
nFAP-non-fed aquaculture in ponds, \\
nFEN-non-fed nearshore aquaculture, \\
PFA-paddy field aquaculture, \\
RAS-recirculating aquaculture systems \\
\hline Box 2. Social, economic, environmental, and resource criteria for sustainable \\
$\quad$ development
\end{tabular}




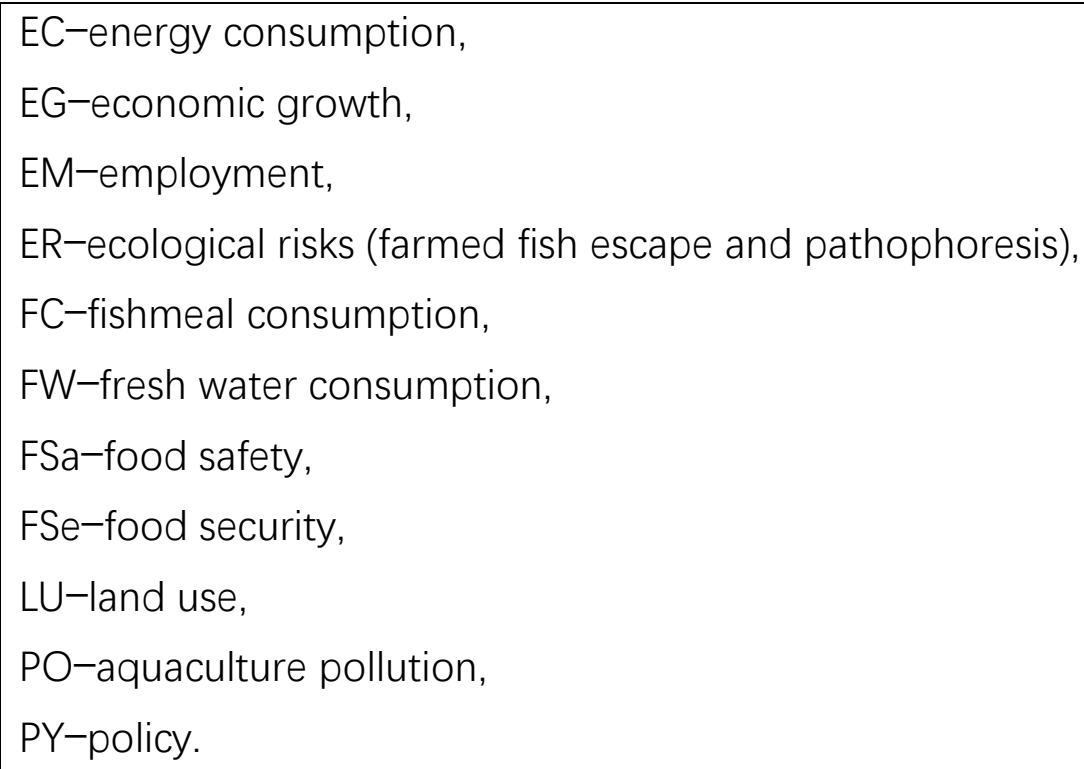

\section{Sustainability evaluation of aquaculture production systems}

The overall sustainability of aquaculture depends on the sustainability of each APSs. Assessing the sustainability of APSs is a multi-criteria decisionmaking problem involving various conflicting criteria (Fig. 2a, S1). Eleven criteria are considered important to this process (Box 2), but only seven of them objective data are available for all ten APSs in China, others are only partially available or immeasurable. Therefore, three methods were used to evaluate the sustainability of the ten major APSs.

Classified aggregation based on objective data. This method calculated the sustainability weights of APSs based on objectively measured criteria of FSe, PO, FC, FW, LU, and EC (Table S1), under the assumption that the criteria have equal weight (see Methods for detail).

The results showed that the ten APSs could be divided into three groups (Fig. 3a). The first group includes nFEN, nFAL, both possess high sustainability weights, greater than 0.10 . The second group includes PFA and nFAP, the sustainability weights of them are between 0.017 and 0.064 . However, the sustainability weights of SALA, FEN, FOA, FAP, RAS and FAL range from 0.0005 to 0.0036 .

Analytical hierarchy process $(\mathrm{AHP})^{24}$. The AHP involves all eleven criteria for all ten APSs (Box 1, Fig. S1), and all the criterion weights used are based on pairwise comparison judgements given by 23 senior and well-trained experts (see Methods). Subsequently, the sustainability weight of each APS was derived 
(Fig. 3b).

The results showed that among eleven criteria the top three (based on their weights with respect to sustainability) in China were PO, EG, FSa (Table S2).

The three non-fed APSs possess large areas, and have several obvious advantages comparing with other APSs (Fig. 4). FEN and FAL possess small areas, and have obvious weaknesses. The sustainability weights of current nFEN, $n F A L, n F A P, P F A$ and FOA are all greater than 0.10 , while those of FAL, FAP, and FEN are all less than 0.08, and RAS and SALA rank in between (Fig. 3b).

AHP partially based on objective data. This method is an AHP involving seven objectively measured criteria, but their own weights with respect to sustainability are determined by pairwise comparison judgements given by the 23 experts mentioned earlier (see Methods).

The results showed that the ten systems could be divided into two groups (Fig. 3c). The APSs of nFEN, nFAL and nFAP belong to one group, each of which possesses a sustainability weight of greater than 0.10. The other seven APSs belong to another group, with sustainability weights of less than 0.07 .

The classified aggregation method based on objective data was an objective method if enough objectively measured criteria were available. On the contrary, the AHP based on experts' judgments is a good option in the absence of available objective data, especially when those unavailable or immeasurable criteria, such as food safety, ecological risk, and policy, are considered. The third method is a combination of the former two methods, in which not only available objective data are used but also the concept of "ecology first" has been considered through experts' judgements. Unfed or almost unfed APSs, i.e. nFEN, nFAL, nFAP, and PFA are considered ecologically friendly systems ${ }^{3,4,7,9,23}$, and they all obtained high sustainability weights by the three methods (Fig. 3).

Although China's aquaculture is considered as one of the most ecologically efficient food production systems in the world, there were only $44.1 \%$ of farmed seafood in 2018 derived from top four APSs with high sustainability weights, and all other APSs had major defects (Fig. 4). Due to the increasing seafood demand and the environmental costs of the simplistic intensification of aquaculture systems, an approach of ecological intensification of aquaculture systems (ELIAS) is proposed to improve the sustainability of China's APSs. 


\section{Ecological intensification of aquaculture systems (ELIAS)}

ELIAS is an approach to improve the production and comprehensive benefits of APSs through rationally integrating anthropogenic inputs with aquaculture ecosystem services. ELIAS stresses trade-offs among various considerations, and aims to produce seafood efficiently while protecting the environment, conserving natural resources, ensuring food security and safety, and promoting social and economic development.

Ecosystem services are defined as processes or conditions that lead to benefits for humans ${ }^{27}$, and are grouped into four categories, i.e. provisioning services, regulating services, supporting services, and cultural services. Due to the high diversity of APSs, various ecosystem services can be integrated into aquaculture ecosystems to improve their outputs and benefits. As in the agriculture ecosystem, some ecosystem services, such as trophic synergism, mutualism, etc., are imperative for improving the sustainability of APSs ${ }^{28-30}$. Additionally, the integrated use of those ecosystem services, such as land space and water for irrigation, is another way to improve the economic benefits of aquaculture ecosystems (Fig. 2b).

The history of aquaculture development is just an evolutionary process of ELIAS, in which the intensification and ecologicalization processes take place alternately with the increasing demand for seafood, advancement of technology, and increasing environmental concerns ${ }^{31,32}$.

ELIAS of recirculating aquaculture system. RAS is a highly intensive APS, and offers several advantages over conventional aquaculture systems, such as reducing water use, land conservation, enhancing feed efficiency, and improving biosecurity $^{4,23}$. However, most RASs nowadays are highly artificial, not taking advantage of many possible ecosystem services, i.e. photosynthesis. Therefore, it is expensive to run, and their products have a much higher carbon footprint ${ }^{4,33}$. Therefore, current RAS is a less sustainable system based on objective criteria in China (Fig. 3).

Comparing with developed countries, China's higher energy cost and relatively lower labor cost make the products from RAS uncompetitive in international and even in local markets. To overcome the barriers of higher operation costs of dissolved waste removal, RAS should be integrated with ecosystem services, like nutrient recovery through photosynthesis of aquatic 
plants, in situ or ex situ ${ }^{34,35}$ (Fig. 2). Aquatic plants integrated in RAS can absorb inorganic nitrogen, release dissolved oxygen, and may serve as a commercial product (e.g., edible seaweeds, vegetables or fruits).

To make RAS profitable and sustainable, conventional RAS can also run with the help of other ecosystem services from the nature or another industry: leveraging alternative sources of energy, such as thermal drainage from power plants or geothermal water. Moreover, non-fossil energy can also be adopted to reduce carbon footprint of RAS.

235 ELIAS of fed aquaculture in ponds. FAP is currently the dominant aquaculture system in many countries of the world. As much as $41.2 \%$ of the total aquaculture production in China was produced in FAP in 2018, mainly in freshwater environment (Fig. 1b). However, FAP is a less sustainable APS (Fig. 3) due to the constrains from PO, FW, FSa and $\mathrm{LU}^{36}$ (Fig. 4C).

FAP involves a vast number of smallholders or family farms from inland to coastal areas. In the future, commercialized integrated aquaculture with an allowable amount of waste discharge should be encouraged to develop. Integrating ecosystem services (e.g., trophic synergism, mutualism, commensalism) into FAP systems (Fig. 2b) can improve pond yields, reduce the discharge of organic matter, and improve food safety and sustainability of pond systems $^{37-39}$. For example, by exploiting synergies between species in polyculture systems the production can be doubled without increasing environment impacts of waste discharge ${ }^{40}$. In addition, some novel intensified integrated models, such as "raceways in pond" and "partitioned aquaculture system", may resolve the issue of operational complexity ${ }^{23}$.

251 ELIAS of fed aquaculture nearshore. FEN is one of the leading opportunities for Chinese fishermen whose employment has undergone a transition from capture fisheries to aquaculture. However, crowded net cages nearshore will cause eutrophication, diseases, and other problems ${ }^{4,33,41}$. Therefore, the sustainability of FEN is very low in China (Fig. 3). Although FEN production is not substantial (Fig. 1b), its high growth rate and negative environmental effects have drawn considerable public attention.

To satisfy the regulations for environmental protection, a large portion of the current FEN should be moved to more exposed areas or offshore areas. Meanwhile, as a stopgap measure, FEN should be integrated with nFEN to 
implement integrated multi-trophic aquaculture (IMTA) and to reduce its negative effects to the maximum extent possible ${ }^{39,42,43}$. Moreover, trade-offs among various stakeholders should be considered in informed planning based on ecological and social carrying capacities ${ }^{44}$.

Offshore aquaculture. An alternative strategy for managing land and water scarcity and waste accumulation is to move aquaculture activities offshore ${ }^{4,45}$. Offshore mariculture can make good use of the physical and biological selfpurification functions (dilution and microbial degradation), which are important marine ecosystem services (Fig. 2b).

FOA in China is defined statistically as mariculture located in sea areas where water depths are $>20 \mathrm{~m}$. Although FOA contributed only to $0.3 \%$ of China's total aquaculture production in 2018, its annual growth rate over the past decade was $15.7 \%$ (Fig. 1b) due to the high-quality seafood from FOA and encouragements from the central and local governments. One operation of FOA in China is the "Deep Blue 1" project for farming salmonids $230 \mathrm{~km}$ southeast off the Qingdao coast in the Yellow Sea (Photo 1).

The sustainability of FOA is considered medium among APSs in China (Fig. 3). FOA should be designed carefully to address environmental concerns associated with conventional FEN, including among others the risks of gene contamination from escaped fish breeding with native (wild) fish and disease transmission ${ }^{4}$. Furthermore, the carrying capacity of FOA within a certain area and suitability of local habitat should be carefully investigated during the planning phase to reduce potential long-term negative environmental effects. Moreover, IMTA and non-fossil energy should be adopted to reduce its environment influences and carbon footprint from $\mathrm{FOA}^{39,42,43,45,46}$.

ELIAS of non-fed APSs. Non-fed APSs include farming filter-feeders (e.g. filterfeeding fishes, bivalves) and some culture-based fisheries ${ }^{47}$ in reservoirs, lakes, and nearshore waters. This category of APSs, including nFAL, nFAP, nFEN, and parts of PFA, is an ecologically efficient and highly sustainable form of APSs (Fig. 3).

In terms of volume, nFEN is the second largest APS in China (Fig. 1b), and is regarded as a carbon sequestration aquaculture system ${ }^{30,48}$. Considering the crucial roles of ecosystem services in these systems, stocking suitable species and optimal quantities of seed (spores, spat, post larvae, fry or fingerlings) 
according to the compositions and productivity of natural food sources (Fig. 2b) are the most economically feasible ways for improving their yields. Moreover, informed planning is needed based not only on physical and production carrying capacities, but also on ecological and social carrying capacities ${ }^{44}$. Integration of aquaculture with agriculture. Paddy field aquaculture (PFA) is a conventional household integrated aquaculture system in many countries, which allows fish and rice to share water and land space (Fig. 2b), and can increase both aquaculture and agriculture yields through mutually beneficial effects between fish and rice shoots ${ }^{5,25,49}$. However, disproportionate fish farming and rice planting within a paddy field may lead to waste accumulation and discharge. Conventional household PFA needs to be scaled up, and commercialized. Moreover, pesticides need to be banned to ensure the food safety of PFA. 3 million hectares of them can be reclaimed to pond-terrace systems (Photo 2) by "Digging pond and raising land" model as it is called in China or "Land shaping model" in India ${ }^{50}$. By the model (i.e. SALA) not only the vast discarded land resources have been exploited, but also aquaculture and agriculture have formed a mutual beneficial development pattern. However, attention should focus on the resalinization of the reclaimed land with the development of SALA due to freshwater consumption. Therefore, farming salt-tolerant aquatic species, such as penaeid shrimp, and application of freshwater conservation techniques should be implemented to reduce fresh water consumption.

Ecosystem services, such as space and cultural services, of aquaculture ecosystems can also be used to magnify the economic benefits of APSs. Integrating aquaculture with tourism, education activity, game fishing, solar power generation (Photo 3) or wind turbines can improve the economic efficiency and employment level of aquaculture systems $s^{30,51}$.

\section{Conclusions and suggestions}

China's aquaculture production has grown at an annual rate of $7.5 \%$ over the past 30 years mainly by simplistic intensification of APSs, which result in higher farming costs and more potential environmental risks in the longer term. Their sustainability can be improved by ELIAS through the following routes in general: 
- For those non-fed "extensive" APSs with less anthropogenic inputs and higher sustainability weights, reasonably stocking of multi-species based on the compositions and productivity of natural food sources in the farming waters is a feasible way of boosting output or "intensification".

- For highly intensive APSs with low sustainability weights, such as RAS, photosynthesis of aquatic plants and other natural services are suggested to be integrated into the systems to reduce the high operation cost of dissolved waste removal and high energy consumption.

- Integrating aquaculture with agriculture in PFA and SALA, moving aquaculture activities offshore (FOA) are imperative to expand aquaculture space scale, which can make good use of mutually beneficial effects between aquaculture and agriculture, and the physical and biological self-purification functions of the seas.

- Integrating aquaculture with tourism, education activity, game fishing, solar power generation or wind turbines can improve the economic efficiency and employment level of aquaculture systems.

At the current stage, the government may formulate and implement a detail targeted development plan according to the sustainability of various farming systems, initiating measures such as

- Establishing partnership with qualified third-party certification bodies to certify farmed seafood from $\mathrm{nFEN}, \mathrm{nFAL}$, and $\mathrm{nFAP}$, which are in line with the rules of organic aquaculture ${ }^{52}$, as organic products so as to promote the development of these farming systems.

- Coordinating the interest relationship among various stakeholders in or along large inland waters or nearshore areas based on ecological and social carrying capacities of aquaculture ${ }^{44}$, so that nFEN and nFAL can develop in an orderly manner.

- Providing preferential policies in terms of land or sea area use, and loans to promote the expansion of SALA, PFA and FOA.

- Phasing out FAL to protect water quality of large inland waters.

- Providing preferential loans for farmers to adopt eco-friendly technics while implementing stringent environmental protection policies to 

promote the ecological intensification of FAP 、FEN and RAS.

- Encouraging the integration of aquaculture activities with green power generation to pursue the goals of high productivity, zero waste discharge and less carbon footprint of aquaculture, simultaneously, in near future.

To achieve the sustainable development of aquaculture in China it is essential for scientists to develop innovative technology, to clarify the ecological and social carrying capacity of specific farming waters or area, to popularize ELIAS knowledge, and so on.

Realization of ELIAS needs close collaboration among policymakers, scientists, farmers, and the supporting industry. Further advances in breeding ${ }^{18}$, feed development (de-linking aquaculture feeds from wild fish and terrestrial plant ingredients) ${ }^{19}$, and disease prevention ${ }^{17}$ will ensure a solid underpinning for achieving the sustainable development of the aquaculture industry in China and in the world.

Due to the intensification trend of the world aquaculture systems and the absence of many objectively measured criteria related to the sustainability of aquaculture and other animal farming systems ${ }^{16}$, our present study is vital for sustainable development of aquaculture in other countries and other animal farming systems.

\section{Materials and methods}

Building the metric data of APSs related to system sustainability. The data on aquaculture production and areas for inland aquaculture waters of mainland China were collected from China Fishery Statistical Yearbook. http://data.cnki.net/Trade/yearbook/single/N2018120050?z=Z009.

The average major species group conversion factors used for converting whole live weight aquatic food product data to edible aquatic meat were as follows: fish 1.15 (gutted, head-on), crustaceans 2.80 (tails/meat, peeled), and molluscs 6.0 (meat, without shells) ${ }^{53}$.

The data of discharge coefficients of pollution sources in China's aquaculture were taken from Handbook of the First National Census of Pollution Sources $^{54}$. The average discharge coefficient was weighed one of 3 or 6 dominant aquatic products in each system (Table S1). 
Estimated average species group economic feed conversion ratios (total feed fed/total species-group biomass increase) were collected from Tang et al. $(2016)^{55}$. The portion of fishmeal and oil in feeds of a specific species was taken from a previous publication ${ }^{56}$. The share of fishmeal and oil consumption of each system was calculated from the weighed value of the dominant species in each aquaculture system (Table S1).

Total water footprint of freshwater species and the water footprint embraced in feed of mariculture species were taken from the previous publications $^{57-59}$. Total freshwater footprint of each system was calculated from the weighed value of the dominant species in each aquaculture system.

For calculating land use for pond farming and RAS, the ratio of 1:1.5 (water area of ponds to total land use for the farm) and 1:10 (water area of tanks to land use for the RAS farm) was applied, respectively ${ }^{60}$.

Energy consumption $(\mathrm{kwh} / \mathrm{kg}$ ) of aquatic products from different aquaculture systems in China was collected or calculated based on a Xu et al. $(2011)^{61}$.

Calculation of sustainability weights. Sustainability evaluation of various APSs is a multi-criteria decision-making problem involving various conflicting criteria. There are eleven criteria contributing to APSs sustainability, but only seven of them have available objective data in China. Therefore, three methods were comparatively proposed to deal with the problem.

i. Classified aggregation based on objective data. This method calculated the sustainability weights of APSs based on the objective data under the assumption that the seven criteria are equally weighted. Seven criteria were available (Table S1) to construct the decision hierarchy. They were classified into three dimensions: Social \& economic, including FSe and EG; Environment, including Po and EC (related to $\mathrm{CO}_{2}$ emission); Resource, including FM, LU and FW.

Before the calculation of local weights under each criterion, the original data may need to be preprocessed. For the criterion under which there are negative values, the original data were transformed into nonnegative values using a minmax transformation under this criterion. If there are zero values that will be applied geometric mean or reciprocals under some criteria, the data were transformed by adding a small ad hoc constant (e.g. 0.0001) into each original data ${ }^{62}$. Moreover, 
note that the values under different criteria represent different meanings. For some criteria, a higher value is better, while for the others, lower is better. The former is referred to as direct criteria and the latter is referred to as inverse criteria. When linear utilities are assumed, the ratio method can be used to transform the actual values into weights ${ }^{63}$. Let $a_{i j}$ be the actual value of an alternative $j$ and $p_{i j}$ be its local weight under criterion $i$. For a direct criterion $i$ (e.g., FSe or EG), the local weight of the alternative $j$ (e.g., aquaculture production system) is given by

$$
p_{i j}=\frac{a_{i j}}{\sum_{j} p_{i j}}
$$

For an inverse criterion $i$ (e.g., Po, EC, FM, LU or FW), the local weight of the alternative $j$ is given by

$$
p_{i j}=\frac{1 / a_{i j}}{\sum_{j} 1 / a_{i j}} .
$$

In our case, linear utilities are assumed, and the local weights of ten APSs under each criterion were calculated using the above ratio method. If the assumption of linear utilities is not valid, or essentially there is lack of related data, weights can be given by experts' judgements.

The last step is synthesis. The most appropriate approach should be identified to compute the aggregate score of each APS based on the above local weights. For this, two relevant rules are offered: if the different dimensions are expected to be compensatory or the different criteria within one dimension are highly correlated with each other, an arithmetic mean is sufficient to calculate the aggregate score; otherwise, a geometric mean can be used for the aggregation ${ }^{64}$. In our case, the criteria within three dimensions appear to be with low internal correlations and the three dimensions are supposed to be almost noncompensatory, therefore geometric means were used for their aggregations.

Under the assumption that the seven criteria are equally weighted, the aggregate score of the $j$ th APS was given by

$$
s_{j}=\left(p_{F S e, j}^{1 / 2} p_{E G, j}^{1 / 2}\right)^{2 / 7}\left(p_{P o, j}^{1 / 2} p_{E C, j}^{1 / 2}\right)^{2 / 7}\left(p_{F M, j}^{1 / 3} p_{L U, j}^{1 / 3} p_{F W, j}^{1 / 3}\right)^{3 / 7}, j=1, \ldots, 10,
$$

where $p_{i j}$ is the local priority of the $j$ th APS under criterion $i$.

Furthermore, the sustainability weight of the $j$ th APS (denoted by $s p_{j}$ ) was calculated by normalizing the aggregate scores of them, i.e.

$$
s p_{j}=\frac{s_{j}}{\sum_{k=1}^{10} s_{k}}, j=1, \ldots, 10,
$$


which can be used for the sustainability ranking.

ii. Analytical hierarchy process (AHP). This method is the analytical hierarchy process $(\mathrm{AHP})^{24}$, which involves all the eleven criteria, however, all local weights used are based on pairwise comparison judgements given by experts. The decision problem is firstly structured as a three-level hierarchy (Fig. S1). The top level is the overall goal of sustainability of APSs. All the eleven criteria (Box 2) contributing to the overall goal are represented in the intermediate level. The lowest level comprises the ten APSs (Box 1) which will be evaluated in terms of the criteria in the intermediate level.

As for some criteria (e.g., food safety, policy, etc.) no data are unavailable or can be measured, the relative importance of various elements in the same level with respect to the elements in their upper level was compared pairwise by the 23 invited senior, well-trained Chinese experts from related fields. The invited experts include seven members of the Fisheries Group of Discipline Assessment Organization of the State Council of China, ten members of the Preparatory Committee of Chinese Society of Aquaculture Ecology, and six senior experts from disciplines of fisheries management, fisheries economics, seafood safety, and aquaculture engineering, as well as related administrators. Their names are listed in the Acknowledgements.

In the intermediate level, a pairwise comparison matrix of eleven criteria with respect to the overall goal was given by aggregating the 23 experts' judgements through geometric mean. The consistency ratio of the matrix is 0.0175 . Therefore, the estimation of the eigenvector $v=\left(v_{1}, \ldots, v_{11}\right)$ associated with the principal eigenvalue was accepted. Then the local weight (Table S2) of the $i$ th criteria with respect to the overall goal was given by

$$
w_{i}=\frac{v_{i}}{\sum_{k=1}^{11} v_{k}}, i=1, \ldots, 11 \text {. }
$$

In the lowest level, each APS has a numerical value for each criterion, which is the geometric mean of the 23 experts' judgements. The local weights of ten systems with respect to each criterion were calculated by normalizing their values under the same criterion. Formally, the local weight (Fig. 4) of the $j$ th APS with respect to the $i$ th criterion was given by

$$
p_{i j}=\frac{b_{i j}}{\sum_{k=1}^{10} b_{i k}}, j=1, \ldots, 10 ; i=1, \ldots, 11,
$$

where $b_{i j}$ is the numerical value of the $j$ th APS under the $i$ th criterion. 
The sustainability weight of the $j$ th aquaculture system was given by

$$
s p_{j}=\sum_{i=1}^{11} w_{i} p_{i j}, j=1, \ldots, 10 .
$$

498

499

500

501

502

503

504

505

506

507

508

509

510

511

512

513

514

515

Based on the sustainability weights, the sustainability ranking of ten APSs was obtained.

iii. AHP partially based on objective data. This method is proposed to take full advantage of existing information, including the relative importance of criteria based on experts' judgements and seven objective criterion data of ten APSs. The decision problem is also structured as a three-level hierarchy. However, the intermediate level only comprises seven criteria having objective data. In the intermediate level, the weights of seven criteria with respect to the sustainable goal were obtained through a pairwise comparison matrix of seven criteria given by 23 experts' judgements. The consistency ratio of the matrix is 0.0266 and the estimation of the eigenvector $v=\left(v_{1}, \ldots, v_{7}\right)$ associated with the principal eigenvalue was accepted. Then the weight of the $i$ th criterion with respect to the overall goal was given by

$$
w_{i}=\frac{v_{i}}{\sum_{k=1}^{7} v_{k}}, i=1, \ldots, 7
$$

In the lowest level, there are actual data of APSs under the seven criteria. Thus, the local weights of ten APSs under each criterion can be calculated using the ratio method as described in the above classified aggregation method.

The sustainability ranking of ten APSs was obtained based on the sustainability weights of the systems, which were given by

$$
s p_{j}=\sum_{i=1}^{7} w_{i} p_{i j}, j=1, \ldots, 10,
$$

where $p_{i j}$ is the local weight of the $j$ th APS under the $i$ th criterion.

\section{References}

1 Hicks, C. C. et al. Harnessing global fisheries to tackle micronutrient deficiencies. Nature 574, 95-98 (2019).

2 FAO. The State of World Fisheries and Aquaculture 2020 - Sustainability in Action. (Food and Agriculture Organization of the United Nations, 2020).

3 Froehlich, H. E., Runge, C. A., Gentry, R. R., Gaines, S. D. \& Halpern, B. S. Comparative terrestrial feed and land use of an aquaculture-dominant world. Proc. Natl. Acad. Sci. USA 115, 5295-5300 (2018). 
5284 Klinger, D. \& Naylor, R. Searching for Solutions in Aquaculture: Charting a Sustainable

529 Course. Annu. Rev. Environ. Resour. 37, 247-276 (2012).

5305 Little, D. C., Newton, R. W. \& Beveridge, M. C. Aquaculture: a rapidly growing and significant source of sustainable food? Status, transitions and potential. Proc. Nutr. Soc. 75, 274-286 (2016).

534 Nyström, M. et al. Anatomy and resilience of the global production ecosystem. Nature 575, 98-108 (2019).

536 Tilman, D., Balzer, C., Hill, J. \& Befort, B. L. Global food demand and the sustainable intensification of agriculture. Proc. Natl. Acad. Sci. USA 108, 20260 (2011).

541 Henriksson, P. J. G., Belton, B., Murshed-e-Jahan, K. \& Rico, A. Measuring the potential for sustainable intensification of aquaculture in Bangladesh using life cycle assessment. Proc. Natl. Acad. Sci. USA 115, 2958-2963 (2018). Cao, L. et al. China's aquaculture and the world's wild fisheries. Science 347, 133-135 (2015).

Damerau, K., Waha, K. \& Herrero, M. The impact of nutrient-rich food choices on agricultural water-use efficiency. Nat. Sustain. 2, 233-241 (2019). Bush, S. R. et al. Certify Sustainable Aquaculture? Science 341, 1067-1068 (2013). Roheim, C. A., Bush, S. R., Asche, F., Sanchirico, J. N. \& Uchida, H. Evolution and future of the sustainable seafood market. Nat. Sustain. 1, 392-398 (2018).
Jouffray, J.-B., Crona, B., Wassénius, E., Bebbington, J. \& Scholtens, B. Leverage points in the financial sector for seafood sustainability. Sci. Adv. 5, eaax3324 (2019). Kittinger, J. N. et al. Committing to socially responsible seafood. Science 356, 912-913 (2017).

Smith, M. D. et al. Sustainability and Global Seafood. Science 327, 784-786 (2010). National Academies of Sciences, E., and Medicine. Science Breakthroughs to Advance Food and Agricultural Research by 2030. (The National Academies Press, 2019). Reverter, M. et al. Aquaculture at the crossroads of global warming and antimicrobial resistance. Nat. Commun. 11, 1870 (2020).

Houston, R. D. et al. Harnessing genomics to fast-track genetic improvement in aquaculture. Nat. Rev. Genet. 21, 389-409 (2020).

Cottrell, R. S., Blanchard, J. L., Halpern, B. S., Metian, M. \& Froehlich, H. E. Global adoption of novel aquaculture feeds could substantially reduce forage fish demand by 2030. Nat. Food. 1, 301-308 (2020).

Føre, M. et al. Precision fish farming: A new framework to improve production in aquaculture. Biosys. Eng. 173, 176-193 (2018).

1 Odum, E. \& Barrett, G. W. Fundamentals of Ecology. 5th edn, (Thomson Brooks/Cole, 2005).

Froehlich, H. E., Gentry, R. R. \& Halpern, B. S. Global change in marine aquaculture production potential under climate change. Nat. Ecol. Evol. 2, 1745-1750 (2018).

Tidwell, J. H. Aquaculture Production Systems. (Wiley Blackwell, 2012).

4 Saaty, T. L. \& Vargas, L. G. Models, Methods, Concepts \& Applications of the Analytic Hierarchy Process. 2nd edn, (Springer, 2012).

25 Gui, J.-F., Tang, Q., Li, Z., Liu, J. \& De Silva, S. S. Aquaculture in China: Success Stories and Modern Trends. (Wiley Blackwell, 2018). 
FAO. The State of World Fisheries and Aquaculture 2018 - Meeting the sustainable development goals (Food and Agriculture Organization of the United Nations, 2018).

27 Daily, G. C. Nature's services. Societal dependence on natural ecosystems. (Island Press, 1997).

Bommarco, R., Kleijn, D. \& Potts, S. G. Ecological intensification: harnessing ecosystem services for food security. Trends Ecol. Evol. 28, 230-238 (2013).

29 Kremen, C. \& Miles, A. Ecosystem services in biologically diversified versus conventional farming systems: benefits, externalities, and trade-offs. Ecol. Soc. 17, 40 (2012).

30 Alleway, H. K. et al. The Ecosystem Services of Marine Aquaculture: Valuing Benefits to

31 Costa-Pierce, B. A. Sustainable Ecological Aquaculture Systems: The Need for a New Social Contract for Aquaculture Development. Mar. Technol. Soc. J. 44, 88-112 (2010).

32 Nash, C. E. The history of aquaculture. (Wiley Blackwell, 2011).

33 Ahmed, N., Thompson, S. \& Glaser, M. Global Aquaculture Productivity, Environmental Sustainability, and Climate Change Adaptability. Environ. Manage. 63, 159-172 (2019). Corey, P., Kim, J. K., Duston, J. \& Garbary, D. J. Growth and nutrient uptake by Palmaria palmata integrated with Atlantic halibut in a land-based aquaculture system. Algae 29, 35-45 (2014).

35 Chang, B. V. et al. Investigation of a Farm-scale Multitrophic Recirculating Aquaculture System with the Addition of Rhodovulum sulfidophilum for Milkfish (Chanos chanos) Coastal Aquaculture. Sustainability 11 (2019).

36 Hai, F. I., Visvanathan, C. \& Boopathy, R. Sustainable Aquaculture (Springer International Publishing, 2018).

37 Shuve, H. et al. Survey finds consumers support Integrated Multi-Trophic Aquaculture. Effective marketing concept key. Glob. Aquac. Advocate 12, 22-23 (2009).

Alexander, K. A., Freeman, S. \& Potts, T. Navigating uncertain waters: European public perceptions of integrated multi trophic aquaculture (IMTA). Environ. Sci. Policy 61, 230237 (2016).

39 Knowler, D. et al. The economics of Integrated Multi-Trophic Aquaculture: where are we now and where do we need to go? Rev. Aquac. (2020).

40 Bosma, R. H. \& Verdegem, M. C. J. Sustainable aquaculture in ponds: Principles, practices and limits. Livest. Sci. 139, 58-68 (2011).

41 Troell, M. et al. Does aquaculture add resilience to the global food system? Proc. Natl. Acad. Sci. USA 111, 13257-13263 (2014).

Chopin, T. Progression of the Integrated Multi-Trophic Aquaculture (IMTA) concept and upscaling of IMTA systems towards commercialization. Aquac. Eur. 36, 5-12 (2011).

Sorgeloos, P. Aquaculture: the blue biotechnology of the future. World Aquac. 35, 16-25 (2013).

44 McKindsey, C. W., Thetmeyer, H., Landry, T. \& Silvert, W. Review of recent carrying capacity models for bivalve culture and recommendations for research and management. Aquaculture 261, 451-462 (2006).

45 Lester, S. E., Gentry, R. R., Kappel, C. V., White, C. \& Gaines, S. D. Offshore aquaculture in the United States: Untapped potential in need of smart policy. Proc. Natl. Acad. Sci. USA 115, 7162-7165 (2018). 


\begin{tabular}{|c|c|c|}
\hline 616 & 46 & \\
\hline 617 & & Aquaculture of Seaweeds. (European Cooperation in Science \& Technolog, 2019). \\
\hline & 47 & De Silva, S. S. Culture based fisheries in Asia are a strategy to augment food security. \\
\hline & & Food Secur. 8, 585-596 (2016). \\
\hline 20 & 48 & Tang, Q. S., Zhang, J. H. \& Fang, J. G. Shellfish and seaweed mariculture increase \\
\hline & & atmospheric CO2 absorption by coastal ecosystems. Mar. Ecol. Prog. Ser. 424, 97-104 \\
\hline & & (2011). \\
\hline 3 & 49 & Lu, J. B. \& Li, X. Review of rice-fish-farming systems in China - One of the Globally \\
\hline 2 & & Important Ingenious Agricultural Heritage Systems (GIAHS). Aquaculture 260, 106-113 \\
\hline & & (2006). \\
\hline & 50 & Dagar, J. C., Yadav, R. K. \& Sharm \\
\hline 7 & & (Springer, 2019). \\
\hline & 51 & Willot, P. A., Aubin, J., Salles, J. M. \& Wilfart, A. Ecosystem service framework and \\
\hline & & typology for an ecosystem approach to aquaculture. Aquaculture 512 (2019). \\
\hline & 52 & Lembo, G. \& Mente, E. Organic Aquaculture Impacts and Future Developments: Impacts \\
\hline & & and Future Developments. (Springer, 2019). \\
\hline & 53 & Tacon, A. G. J. \& Metian, M. Fish Matters: Importance of Aquatic Foods in Human \\
\hline & & Nutrition and Global Food Supply. Rev. Fish. Sci. 21, 22-38 (2013). \\
\hline & 54 & China Pollution Source Census. Handbook of the First National Census of Pollution \\
\hline & & Sources - Discharge Coefficient of Pollution Sources in Aquaculture In Chinese \\
\hline & & <https://www.doc88.com/p-037431165661.html> (2009). \\
\hline & 55 & Tacon, A. G. J. \& Metian, M. Feed Matters: Satisfying the Feed Demand of Aquaculture. \\
\hline & & Rev. Fish. Sci. Aquac. 23, 1-10 (2015). \\
\hline & 56 & Tang, Q., Han, D., Mao, Y., Zhang, W. \& Shan, X. Species composition, non-fed rate and \\
\hline & & trophic level of Chinese aquaculture. J. Fish. Sci. China (2016). \\
\hline & 57 & Ouyang, Y. T. Study on water footprint of freshwater cultured fish and spatial \\
\hline & & optimization of cultured policy in China. Master's Thesis thesis, Dalian University of \\
\hline & & Technology, (2018). \\
\hline & 58 & Pahlow, M., van Oel, P. R., Mekonnen, M. M. \& Hoekstra, A. Y. Increasing pressure on \\
\hline & & freshwater resources due to terrestrial feed ingredients for aquaculture production. Sci. \\
\hline & & Total Environ. 536, 847-857 (2015). \\
\hline & 59 & Mohanty, R. K., Ambast, S. K., Panigrahi, P. \& Mandal, K. G. Water quality suitability and \\
\hline & & water use indices: Useful management tools in coastal aquaculture of Litopenaeus \\
\hline & & vannamei. Aquaculture 485, 210-219 (2018). \\
\hline & 60 & Boyd, C. E. \& McNevin, A. A. Aquaculture, Resource Use, and the Environment. (Wiley \\
\hline & & Blackwell, 2014). \\
\hline & 61 & $\mathrm{Xu}, \mathrm{H}$. et al. The research and development proposals on fishery energy saving and \\
\hline & & emission reduction in China. J. Fish. China 410, e21299-e21299 (2011). \\
\hline & 62 & Sironen, S., Seppälä, J. \& Leskinen, P. Towards more non-compensatory sustainable \\
\hline & & society index. Environ. Dev. Sustain. 17, 587-621 (2015). \\
\hline & 63 & Ramanathan, R. \& Ganesh, L. S. Using AHP for resource allocation problems. Eur. J. \\
\hline & & Oper. Res. 80, 410-417 (1995). \\
\hline & 64 & Béné, C. et al. Global map and indicators of food system sustainability. Sci. Data 6 \\
\hline & & (2019). \\
\hline
\end{tabular}


660

661

662

663

664

665

666

667

668

669

670

671

672

673

674

675

676

677

678

679

680

681

682

683

684

\section{Acknowledgements}

This study was funded by Natural Science Foundation of China grant 31572634, NSFC-Shandong Province joint founding project U1906206 and the National Blue Granary S \& T Innovation Program (SQ2019YFD090086). We thank George N Somero and Rosamond L. Naylor for helpful comments on the paper, and thank Binlun Yan, Dapeng Li, Guoxing Nie, Jiashou Liu, Jiting Sun, Jinlong Yang, Jiasong Zhang, Hong Lin, Hui Liu, Pao Xu, Xiangli Tian, Xiaojuan Cao, Xiaojun Yan, Yaoguang Zhang, Ying Liu, Yong Liang, Yuze Mao, Weimin Wang, and Wen Zhao for making their pairwise comparison judgements on relative importance of eleven criteria and sustainability of eleven aquaculture systems.

\section{Author contributions}

S.L.D., Y.W.D., and P.S. developed the initial concept and wrote the abstract. S.L.D. wrote the initial draft, and Y.W.D. created Figures and Tables. The draft was revised and improved by J.V., Y.O., L.C., Y.G.Z., L.L., J.Y.L. and Y.T.M. The analytical hierarchy process (AHP) was done by W.J.L. and Q.Z.F. The final manuscript was edited by P.S.

\section{Competing interests}

The authors declare no competing interests.

\section{Additional information}

Supplementary information is available for this article at $\cdots$ 
Figures
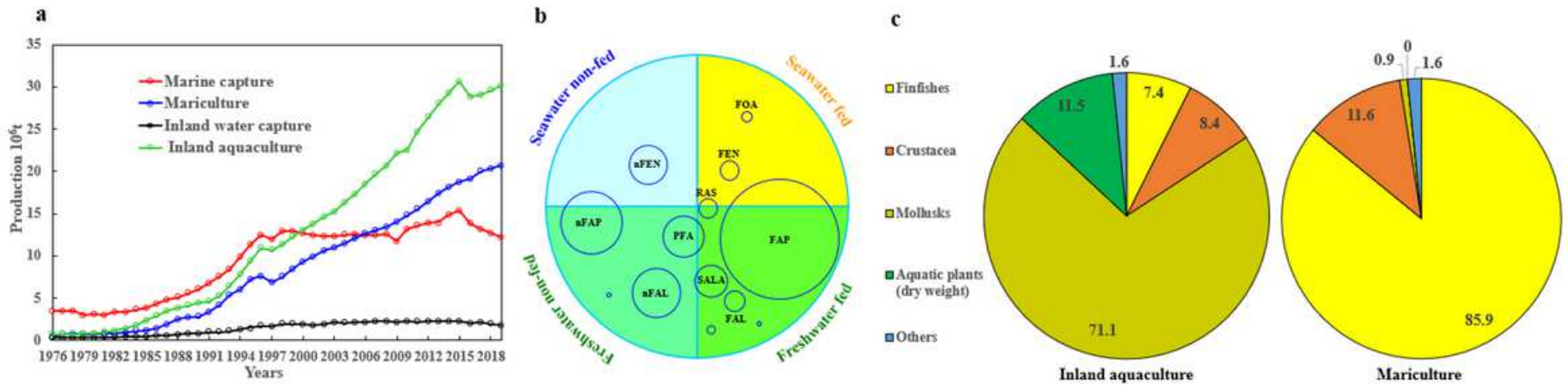

\section{Figure 1}

Status and trends of China's aquaculture in 2018. a, Development trends of fisheries and aquaculture in China mainland. b, Productions of major aquaculture production systems; The area of the circle represents the yield. c, Composition of inland aquaculture and mariculture products.

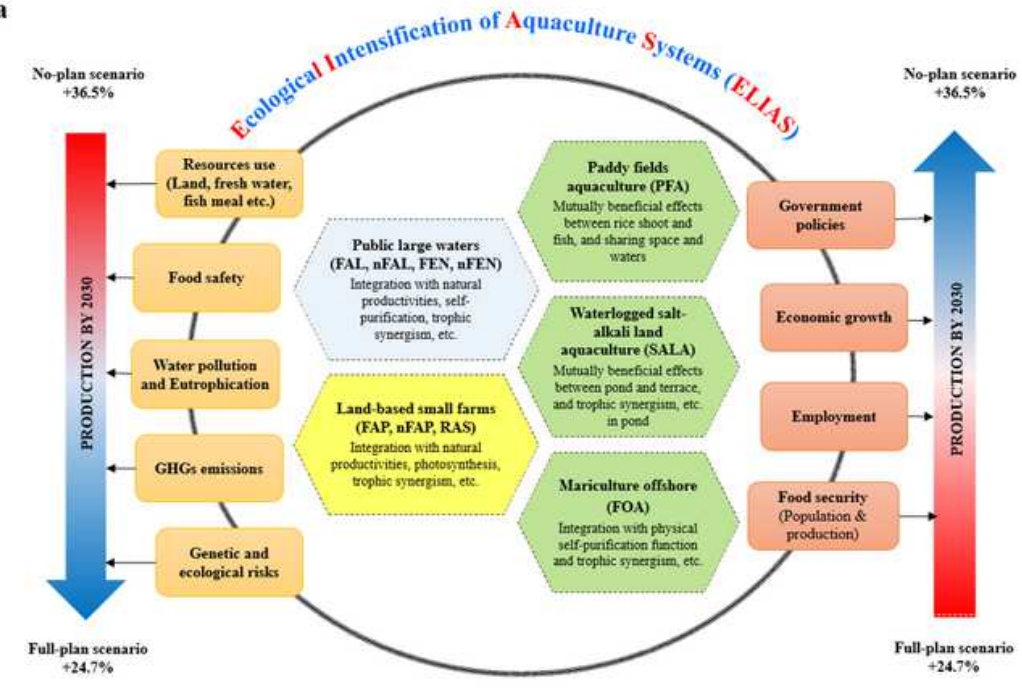

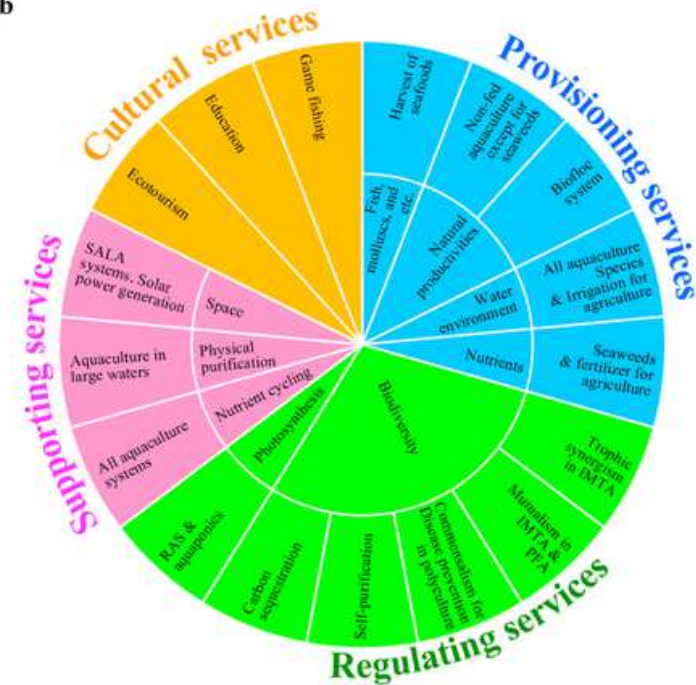

Figure 2

Conceptual framework of ecological intensification of aquaculture systems (ELIAS) and ecosystem services of aquaculture ecosystems. a, Conceptual framework of ELIAS. b, Major ecosystem services of aquaculture ecosystem. 

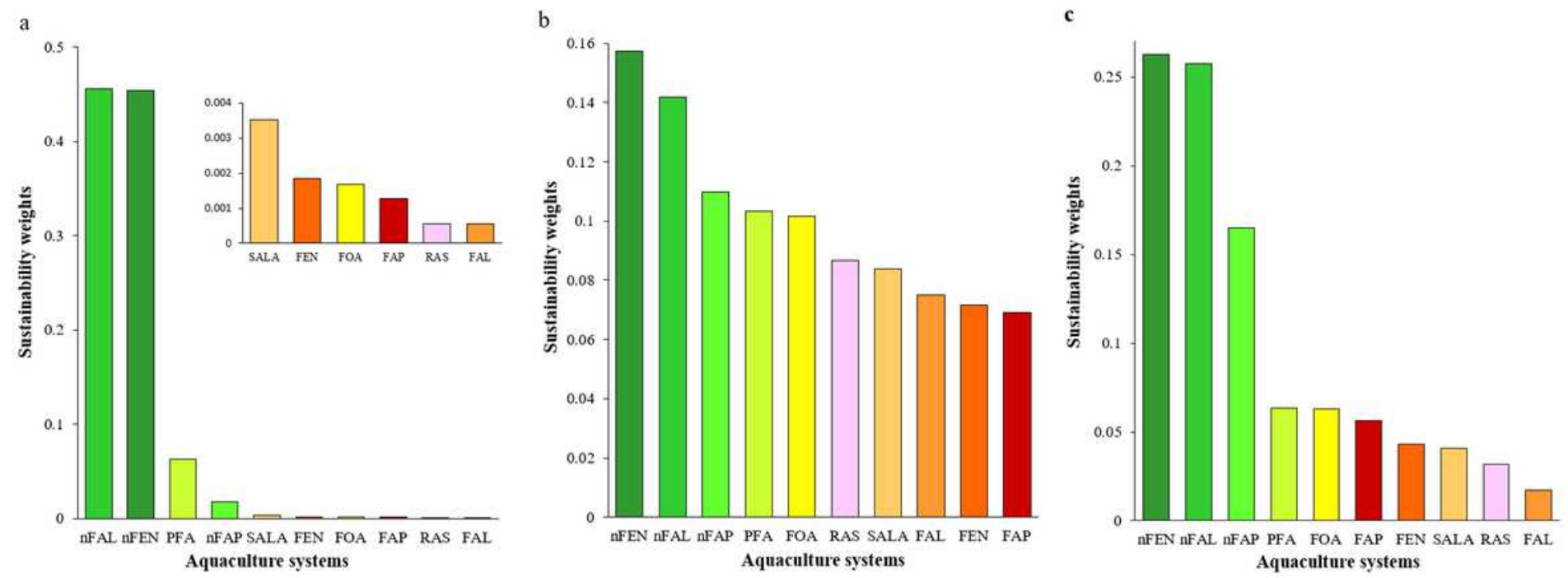

Figure 3

Sustainability weights of major aquaculture production systems in China. a, Evaluation results by Classified aggregation based on objective data. b, Evaluation results by Analytical hierarchy process (AHP). c, Evaluation results by AHP partially based on objective data.
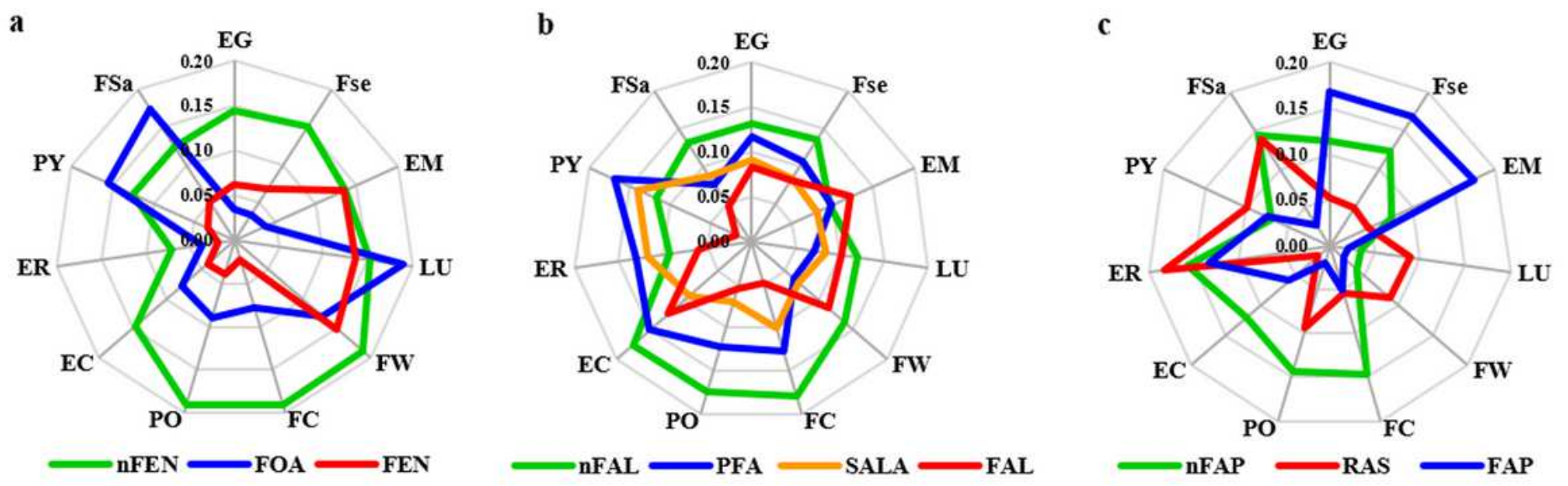

Figure 4

Economic, social, environmental and resource use characteristics of major aquaculture production systems in China. a, The local weights of nFEN, FOA and FEN with respect to each criterion. $b$, The local weights of $n F A L$, PFA, SALA and FAL to each criterion. $c$, The local weights of nFAP, RAS and FAP to each criterion. 

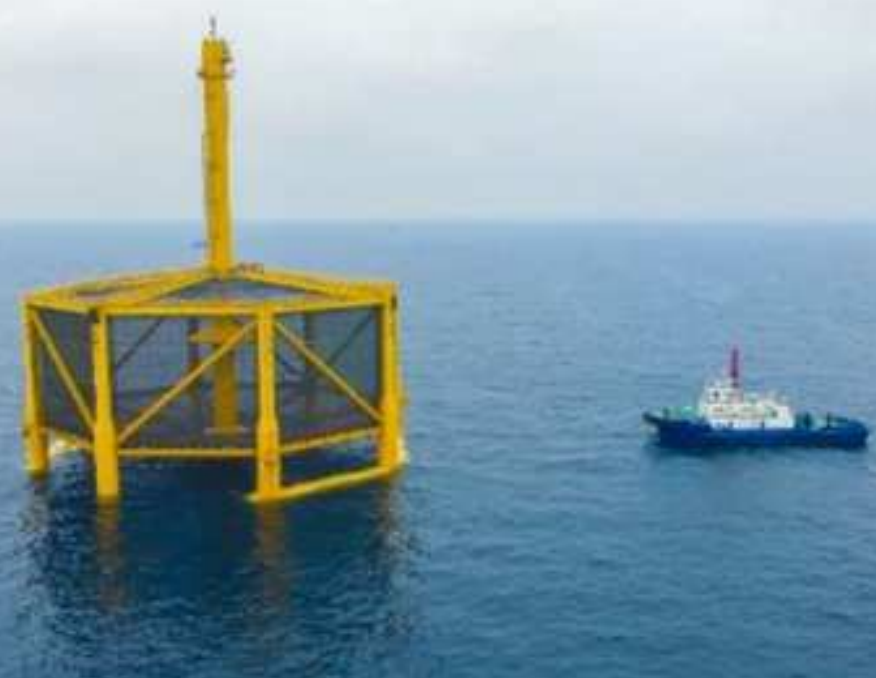

\section{Figure 5}

Photo 1| “Deep Blue 1”, a semi-submersible salmonid farming apparatus located far-offshore.

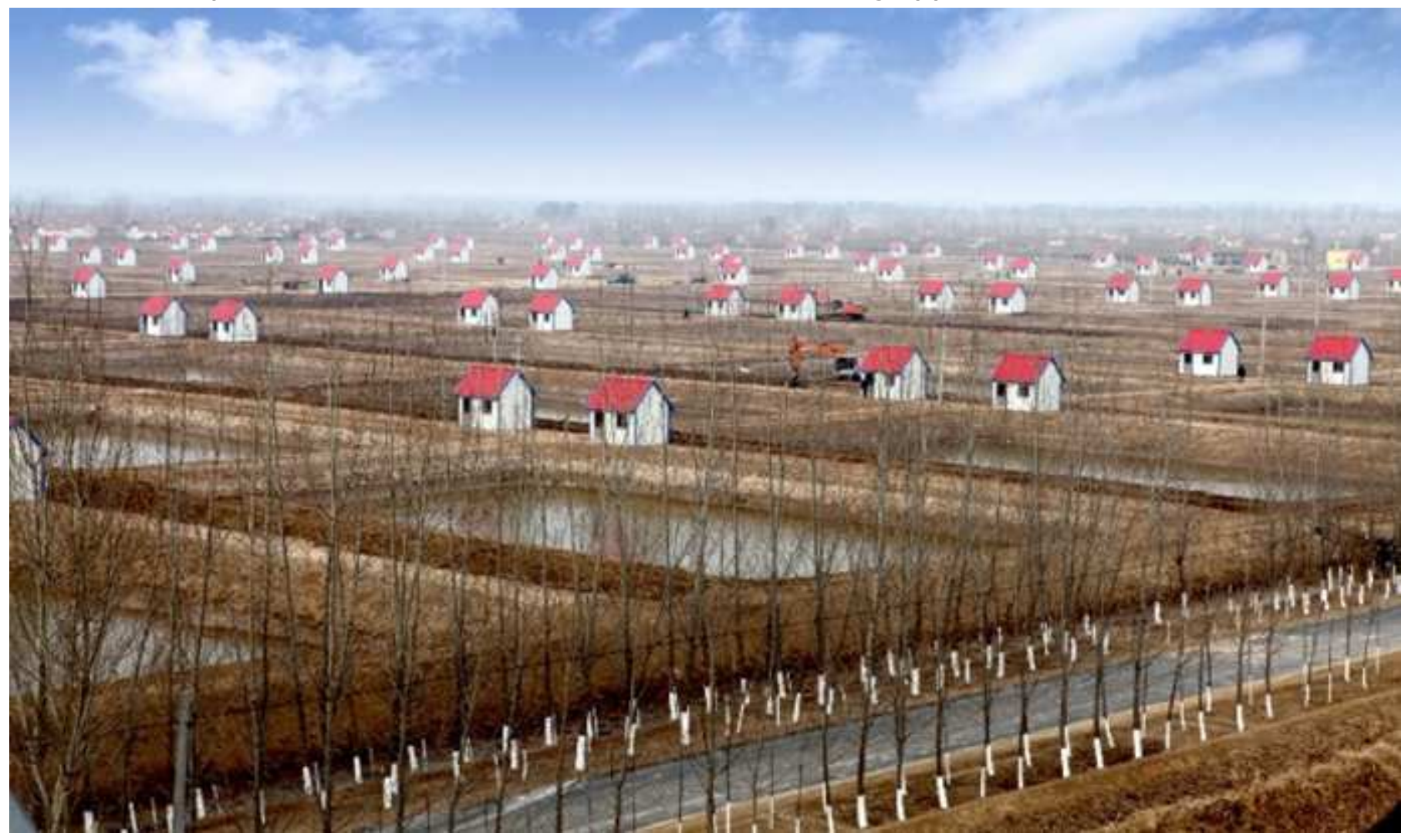

Figure 6 
Photo 2 | Waterlogged salt-alkali land aquaculture (SALA) in China.

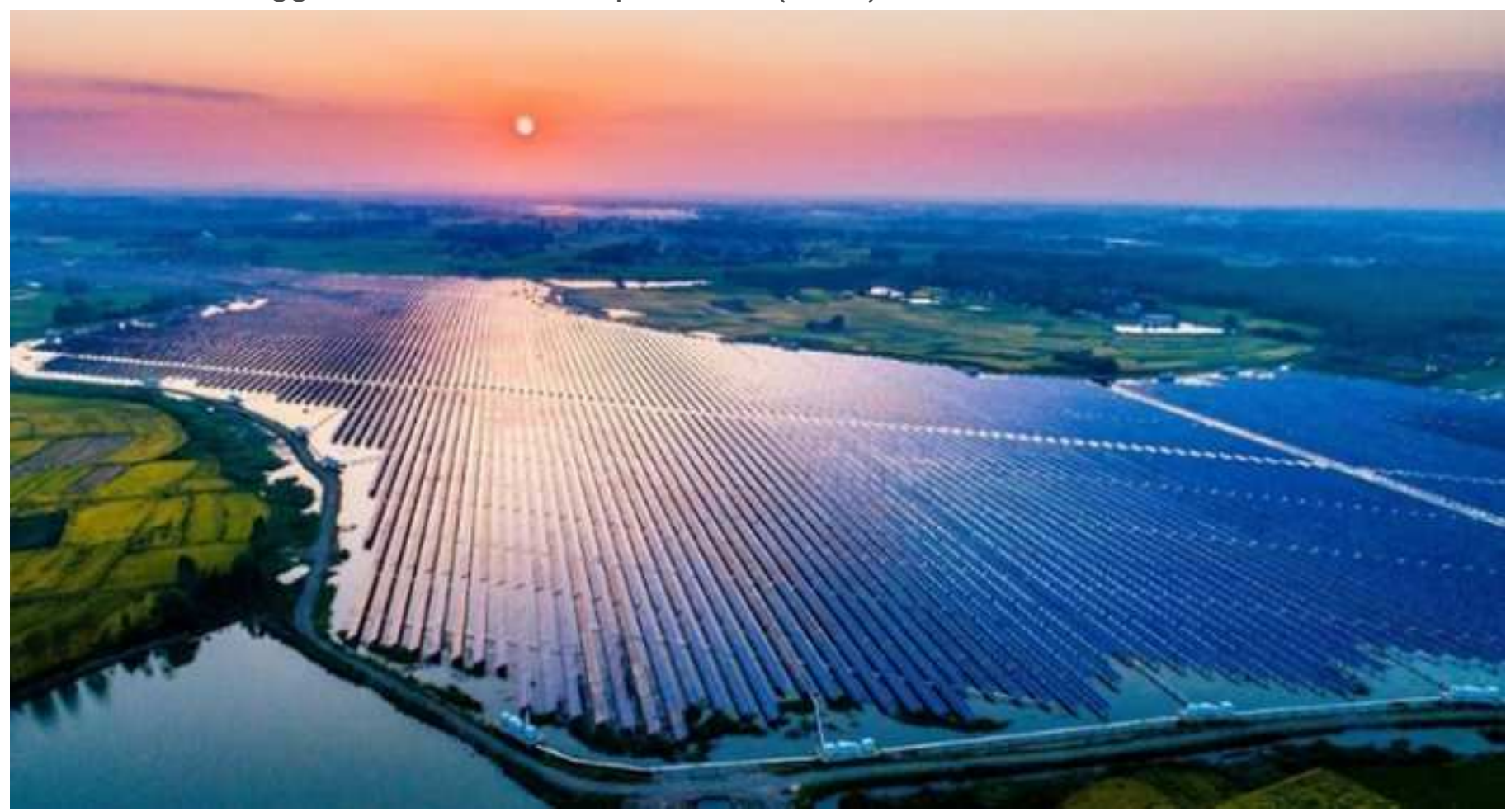

Figure 7

Photo 3 | Integration aquaculture with solar power generation

\section{Supplementary Files}

This is a list of supplementary files associated with this preprint. Click to download.

- Figures1.docx

- Tables1.docx

- Tables2.docx

- Sl.docx 